\title{
Right Anterior Middle Peripheral Zone of Prostate
}

National Cancer Institute

\section{Source}

National Cancer Institute. Right Anterior Middle Peripheral Zone of Prostate. NCI

Thesaurus. Code 128606.

The region of the prostate that is located on the anatomical right side of the anterior portion of the middle division of the peripheral zone. 